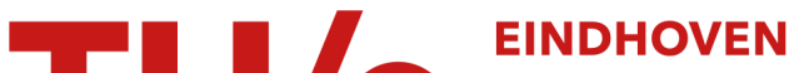 \\ UNIVERSITY OF \\ TECHNOLOGY
}

\section{Field dependence of the thermal conductivity of $\mathrm{CoBr} 2.6 \mathrm{H} 2 \mathrm{O}$}

Citation for published version (APA):

Buys, J. A. H. M., \& De Jonge, W. J. M. (1982). Field dependence of the thermal conductivity of $\mathrm{CoBr} 2.6 \mathrm{H} 2 \mathrm{O}$. Physical Review B, 25(2), 1322-1330. https://doi.org/10.1103/PhysRevB.25.1322

DOI:

10.1103/PhysRevB.25.1322

Document status and date:

Published: 01/01/1982

\section{Document Version:}

Publisher's PDF, also known as Version of Record (includes final page, issue and volume numbers)

\section{Please check the document version of this publication:}

- A submitted manuscript is the version of the article upon submission and before peer-review. There can be important differences between the submitted version and the official published version of record. People interested in the research are advised to contact the author for the final version of the publication, or visit the $\mathrm{DOI}$ to the publisher's website.

- The final author version and the galley proof are versions of the publication after peer review.

- The final published version features the final layout of the paper including the volume, issue and page numbers.

Link to publication

\section{General rights}

Copyright and moral rights for the publications made accessible in the public portal are retained by the authors and/or other copyright owners and it is a condition of accessing publications that users recognise and abide by the legal requirements associated with these rights.

- Users may download and print one copy of any publication from the public portal for the purpose of private study or research.

- You may not further distribute the material or use it for any profit-making activity or commercial gain

- You may freely distribute the URL identifying the publication in the public portal.

If the publication is distributed under the terms of Article 25fa of the Dutch Copyright Act, indicated by the "Taverne" license above, please follow below link for the End User Agreement:

www.tue.nl/taverne

Take down policy

If you believe that this document breaches copyright please contact us at:

openaccess@tue.nl

providing details and we will investigate your claim. 


\title{
Field dependence of the thermal conductivity of $\mathrm{CoBr}_{2} \cdot 6 \mathrm{H}_{2} \mathrm{O}$
}

\author{
J. A. H. M. Buys and W. J. M. de Jonge \\ Department of Physics, Eindhoven University of Technology, 5600 MB Eindhoven, The Netherlands
}

(Received 28 July 1981)

\begin{abstract}
The thermal conductivity of $\mathrm{CoBr}_{2} \cdot 6 \mathrm{H}_{2} \mathrm{O}$ has been measured in the temperature range $1.5-30 \mathrm{~K}$ and in magnetic fields up to $90 \mathrm{kOe}$. The specific field dependence of the data, especially with the field along the easy axis, indicates that the magnons do not contribute to the heat transport, but mainly act as a scattering source for the phonons. The data can be understood qualitatively by confronting them with calculated field-dependent magnon spectra. The actual scattering mechanism seems to be governed by the twomagnon-one-phonon process.
\end{abstract}

\section{INTRODUCTION}

Together with $\mathrm{CoCl}_{2} \cdot 6 \mathrm{H}_{2} \mathrm{O}, \mathrm{CoBr}_{2} \cdot 6 \mathrm{H}_{2} \mathrm{O}$ has been the subject of a large number of investigations ${ }^{1-4}$ because for a long time these compounds were considered to be good physical approximations of the two-dimensional $X Y$ model. The twodimensionality of $\mathrm{CoBr}_{2} \cdot 6 \mathrm{H}_{2} \mathrm{O}$ is clearly visualized by the crystallographic cleavage plane, the $a b$ plane (Fig. 1). The exchange interactions in this plane are supposed to be much larger than the interactions along the $c$ direction. ${ }^{2}$ The dominant exchange interaction in the plane is $J_{1}$. It has been shown ${ }^{2}$ that $J_{1}^{x} \approx J_{1}^{y} \approx-2.4 \mathrm{~K}$ and $-0.7 \mathrm{~K}<J_{1}^{z}$ $<-0.4 \mathrm{~K}$, reflecting the suggested $X Y$ behavior. The resulting antiferromagnetic structure is depicted in Fig. 2(a). In Fig. 2(b) we show the orientation of the three main magnetic axes, i.e., the easy axis $\gamma$, the intermediate axis $\beta$, which coincides

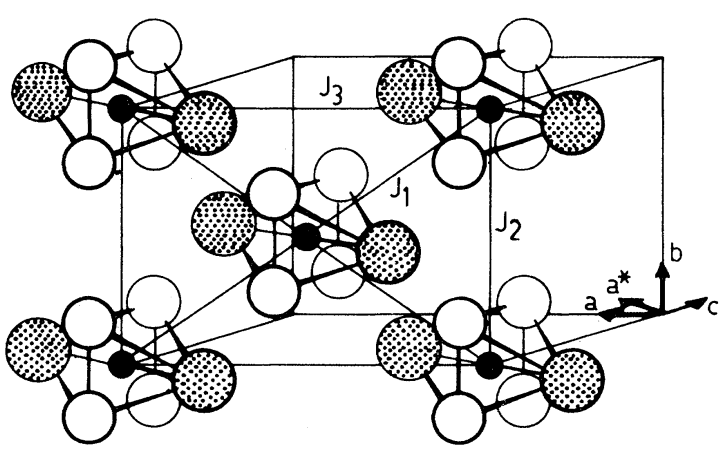

FIG. 1. Part of the crystal structure of $\mathrm{CoBr}_{2} \cdot 6 \mathrm{H}_{2} \mathrm{O}$. Cobalt ions are black, bromine ions are shaded, and oxygen is represented by open circles. $J_{1}, J_{2}$, and $J_{3}$ are the exchange interactions in the $a b$ plane. with the crystallographic $b$ direction, and the hard axis $\alpha$. The experimentally observed phase diagrams of $\mathrm{CoBr}_{2} \cdot 6 \mathrm{H}_{2} \mathrm{O}$ with the external field applied along the easy and intermediate direction are reproduced in Fig. 3. The curves are a combination of the results of Refs. 1 and 3. The dotted curve in Fig. 3 is the anticipated phase boundary with the field applied along the hard direction, where we used the experimental value $g_{a}=2.2$.

Recently it was reported ${ }^{5}$ that the phase diagram corresponding to the easy direction showed some drastic changes when the crystals were partially
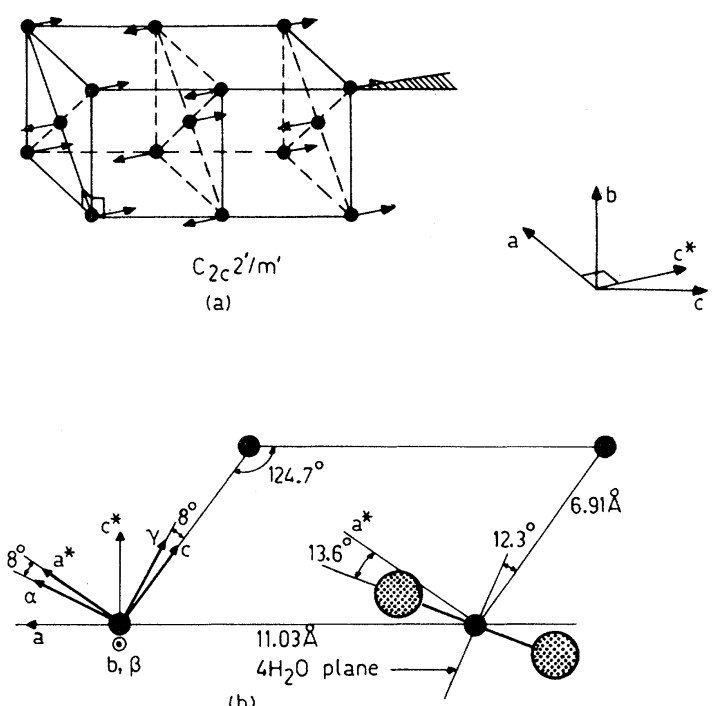

FIG. 2. (a) Magnetically-ordered structure of $\mathrm{CoBr}_{2} \cdot 6 \mathrm{H}_{2} \mathrm{O}$. (b) Location of the easy axis $\gamma$ and the hard axis $\alpha$ in the ac plane. The intermediate axis $\beta$ coincides with $b$. 


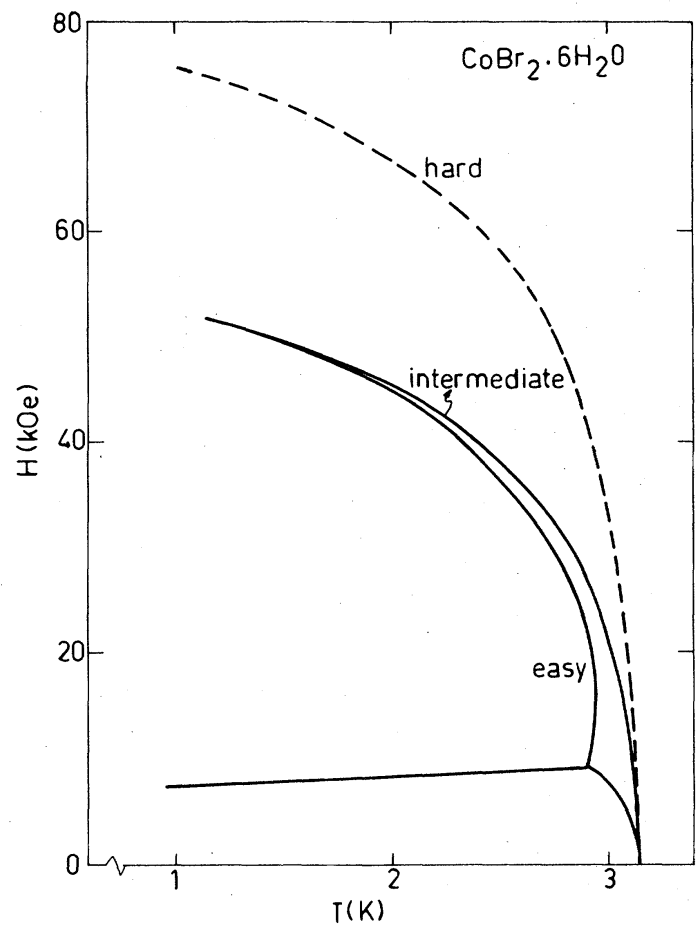

FIG. 3. Magnetic phase diagram of $\mathrm{CoBr}_{2} \cdot 6 \mathrm{H}_{2} \mathrm{O}$ with the external field applied along the three principal directions.

deuterated. In a specimen containing $48 \% \mathrm{D}_{2} \mathrm{O}$ the first-order spin-flop transition line did split up, thus giving rise to a so-called "intermediate state" between the antiferromagnetic and spin-flop region. It is assumed that such an intermediate phase can exist in the presence of a large magneto-elastic coupling. ${ }^{6}$ Such a coupling, which is expected to be of the same strength in $\mathrm{CoBr}_{2} \cdot 6 \mathrm{H}_{2} \mathrm{O}$, may produce large magnetic effects in the thermal conductivity. Moreover, the magnitude of the critical fields, displayed in Fig. 3, allows us to investigate the field and temperature dependence of the thermal conductivity in those regions of the phase diagram where the most drastic changes in the magnetic system occur. The reason why we have chosen $\mathrm{CoBr}_{2} \cdot 6 \mathrm{H}_{2} \mathrm{O}$ instead of $\mathrm{CoCl}_{2} \cdot 6 \mathrm{H}_{2} \mathrm{O}$ or the partially deuterated bromide compound is twofold. First, the experimental setup requires crystals with at least one dimension of about $1 \mathrm{~cm}$, which is most easily realized for $\mathrm{CoBr}_{2} \cdot 6 \mathrm{H}_{2} \mathrm{O}$. Second, both $\mathrm{CoCl}_{2} \cdot 6 \mathrm{H}_{2} \mathrm{O}$ and the partially deuterated bromide compound reveal the quoted intermediate phases. ${ }^{5}$ The excitation spectrum of these magnetic systems is not know in detail, in contrast to spin-flop systems such as $\mathrm{CoBr}_{2} \cdot 6 \mathrm{H}_{2} \mathrm{O}$.

The thermal conductivity of $\mathrm{CoCl}_{2} \cdot 6 \mathrm{H}_{2} \mathrm{O}$ has already been reported in the literature, ${ }^{7,8}$. but these results are restricted to a limited field and temperature range ( $T<4 \mathrm{~K}, H<13 \mathrm{kOe}, H=50 \mathrm{kOe})$. Especially the field dependence needs some extra attention, because the spin-wave interpretation given by Donaldson et al. ${ }^{7}$ is somewhat oversimplified. We shall first discuss the experimental methods which we used to measure the thermal conductivity at low temperatures $(1.3 \mathrm{~K}<T<40$ $\mathrm{K}$ ) and high magnetic fields (up to $90 \mathrm{kOe}$ ). Next, we present our results and give a qualitative interpretation, based on a spin-wave description of the magnetic system and the assumption that magnons merely act as a scattering mechanism for phonons. We shall conclude the paper with a discussion about the nature of the magnon-phonon scattering processes that may be involved.

\section{EXPERIMENTAL METHODS}

The thermal conductivity is measured with a standard steady-state heat-flow method. A known heat-flow $\dot{Q}$ is applied to the crystal, and the resulting temperature difference $\Delta T=T_{2}-T_{1}$ is measured. If $L$ is the distance between the thermometers recording $T_{1}$ and $T_{2}$ and $A$ is the cross section of the crystal, the thermal conductivity is given by

$$
\lambda=\frac{L \dot{Q}}{A \Delta T} .
$$

The main experimental problem is the accurate determination of small-temperature differences at low temperatures. For that reason we shall only give a brief description ' of the experimental apparatus but consider the thermometry in more detail.

The crystal (1) is clamped in a copper crystal holder (4) which is attached to the upper flange (10) of the sample assembly via a copper (5) and a brass (9) bar (see Fig. 4). The evacuated copper can (11) is immersed in a liquid ${ }^{4} \mathrm{He}$ bath. The sample is positioned at the center of a superconducting solenoid. A metal film resistor (2) is used as a heater and attached to the top of the crystal with some GE7031 varnish. A temperaturecontrol unit consisting of a carbon resistor (7) as a sensor, and a manganine heater is used to control the temperature of the crystal holder. The brass bar (9) serves as a thermal resistance in order to obtain temperatures above $4.2 \mathrm{~K}$. A germanium resistor (6) is attached to the copper bar (5) for calibration purposes. 


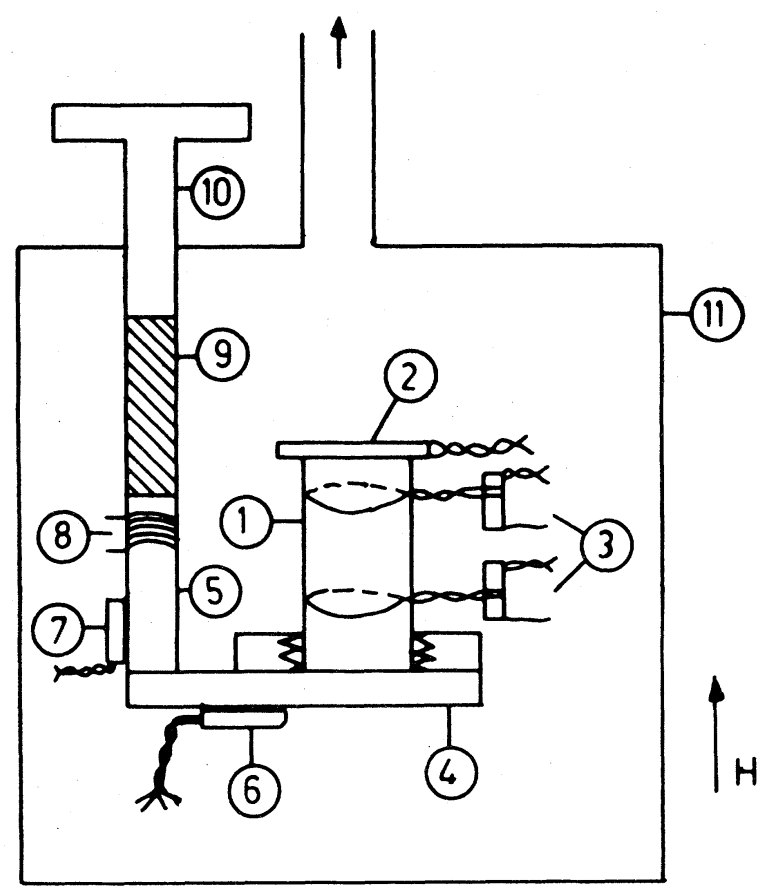

FIG. 4. Sample assembly of the thermal-conductivity measuring system. The orientation of the crystal with respect to the external field can be varied by an alternate connection of the crystal support (4) to the lower brass bar (5).

The temperature difference caused by a heat flow $\dot{Q}$ is measured with two 100- $\Omega$ Allen-Bradley carbon resistors $R_{1}$ and $R_{2}$ (3). Both are thermally anchored to the crystal by means of copper wires. The two resistors can be calibrated against the germanium thermometer $(\dot{Q}=0)$. The zero-field calibration data are numerically fitted to the formula:

$$
\left(\frac{\ln R}{T}\right]^{1 / 2}=\sum_{n=0}^{3} A_{n}\left[(\ln R)^{1 / 2}\right]^{n} .
$$

The magnetoresistance of the carbon thermometers was incorporated by fitting field-dependent calibration data to the formula:

$$
\frac{R(H)-R(0)}{R(0)}=\frac{A H^{2}}{T^{3 / 2}+B H^{2}} .
$$

The coefficients $A$ and $B$ were found to vary approximately linear with temperature. These interpolation formulas described the data of each individual thermometer in the successive temperature ranges $1.2<T<4.5 \mathrm{~K}$ and $4.5<T<9 \mathrm{~K}$ with an accuracy of about $1 \mathrm{mK}$ at zero field and about twice this value at the highest fields. At higher temperatures we used cubic splines to fit the zero- field calibration data. In this temperature region deviations exceed $2 \mathrm{mK}$. This implies thatgenerally speaking-temperature differences can be determined with an accuracy of $2 \mathrm{mK}$ at the lowest temperatures, which would require a temperature difference between $R_{1}$ and $R_{2}$ of at least $100 \mathrm{mK}$ during the experiments in order to gain the desired accuracy of a few percent. As this requirement would severely limit the accessibility of the low-temperature region, we have chosen a socalled two-step measuring method. This method is based on the fact that the error $f(T)=T_{\text {calc }}(R)$ $-T_{\text {expt }}$ of the interpolation formula describing the behavior of a carbon thermometer varies very slowly as a function of temperature $[d f(T) / d T$ is of the order of $\left.10^{-4}-10^{-3}\right]$. Within a restricted temperature region the error may therefore be regarded as an offset with a (maximum) temperature dependence of $10^{-3}$. In the two-step measuring method the (local) value of this offset is implicitly determined.

(a). A heat flow $\dot{Q}$ is applied to the crystal and the desired temperature is set with the aid of the temperature control unit. After both temperature readings have stabilized (typically within a few minutes), $R_{1}$ and $R_{2}$ are measured $\left(R_{1}^{I}\right.$ and $R_{2}^{I}$, respectively).

(b). $\dot{Q}$ is switched off and with the aid of the temperature control unit $R_{1}$ is adjusted to its initial value $R_{1}^{I}$. The thermometer $R_{2}$ now stabilizes at a value of $R_{2}^{F}$.

The temperature difference $\Delta T$ caused by the heat flow $\dot{Q}$ can now be calculated directly:

$$
\Delta T=T_{\text {calc }}\left(R_{2}^{F}\right)-T_{\text {calc }}\left(R_{2}^{I}\right),
$$

or, more conveniently,

$$
\Delta T=\left(\frac{d T_{\text {calc }}}{d R_{2}}\right)_{R_{2}^{a v}}\left(R_{2}^{F}-R_{2}^{I}\right),
$$

where $R_{2}^{a v}$ is defined as

$$
R_{2}^{a v}=\frac{1}{2}\left(R_{2}^{F}+R_{2}^{I}\right)
$$

The value of $\lambda$, which can be evaluated from Eq. (1), is attributed to the average temperature $T^{a v}$ corresponding to $R_{2}^{a v}$.

The essence of the two-step measuring method is the reduction of the error due to fitting procedures. An absolute error of $2 \mathrm{mK}$ in $\Delta T$ is replaced by a relative error equal to $d f(T) / d T$ in $\left(d T_{\text {calc }} / d R_{2}\right)_{R_{2}^{a v}}$. As can be seen in Eq. (5) the ul- 
timate error in $\Delta T$ is a combination of the "calibration" error in $\left(d T_{\text {calc }} / d R_{2}\right)_{R_{2}^{a v}}$ and the experimental error in $R_{2}^{F}-R_{2}^{I}$. This experimental inaccuracy can be minimized by modifying the thermometer resistance bridge in such a way thatinstead of $R_{1}$ and $R_{2}$ - the values of $R_{1}$ and $R_{1}-R_{2}$ are measured. In this way the effect of small temperature variations of the sample assembly as a whole on the determination of $\Delta T$ can be eliminated or suppressed and the full advantages of the two-step measuring method can be exploited. In general, temperature differences $\Delta T$ as small as $0.1 \mathrm{mK}$ could be determined with a relative error of about $1 \%$, the experimental resolution being limited by electrical noise.

\section{RESULTS}

Measurements of the thermal conductivity of $\mathrm{CoBr}_{2} \cdot 6 \mathrm{H}_{2} \mathrm{O}$ were performed with the heat flow $\dot{Q}$ in the $b$ direction. The dimensions of the crystals in the other crystallographic directions were too small to carry out experiments. In Fig. 5 we plotted the thermal conductivity $\lambda$ as a function of temperature for two distinct cases, namely for zero external field and with a field of $90 \mathrm{kOe}$ parallel to the preferred direction of spin alignment $\gamma$. At the lowest temperatures $(T<2.5 \mathrm{~K})$ the high-field data represent the lattice thermal conductivity, since in that temperature and field range magnons can

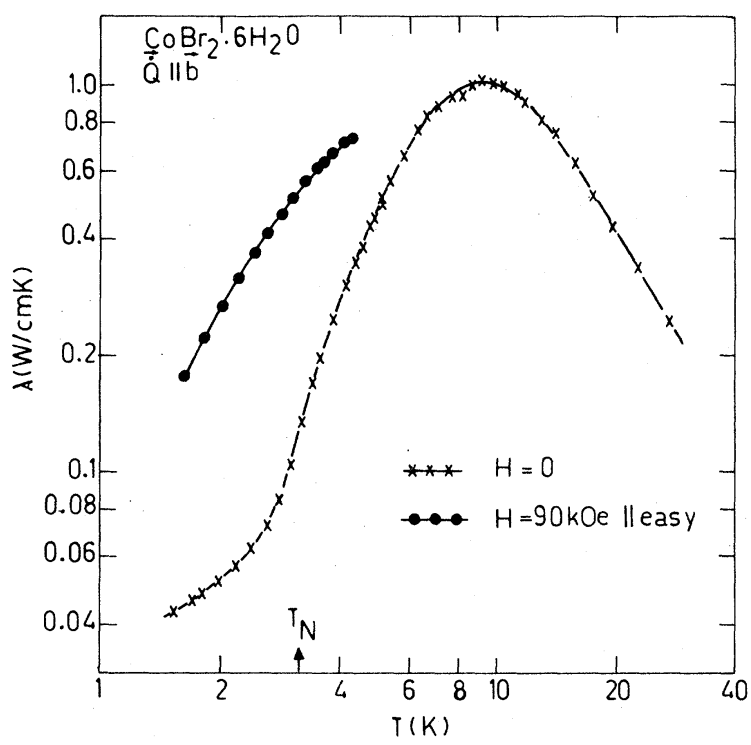

FIG. 5. Temperature dependence of the thermal conductivity of $\mathrm{CoBr}_{2} \cdot 6 \mathrm{H}_{2} \mathrm{O}$ at external fields of 0 and 90 kOe. hardly be excited, as we shall see later on. The limiting low-temperature behavior of the high-field curve can give some information about the dimensionality of the lattice system. In Fig. 5 we find $\lambda$ $(90 \mathrm{kOe}) \sim T^{2.0}$ for $T<2.5 \mathrm{~K}$. One might be tempted to ascribe two-dimensional characteristics to the lattice system. The lattice specific heat, ${ }^{2}$ however, is proportional to $T^{3}$ below $11 \mathrm{~K}$, and hence we expect this three-dimensional behavior in $\lambda(90 \mathrm{kOe})$ at temperatures below $1.5 \mathrm{~K}$, indicating the influence of various scattering processes on $\lambda$.

The zero-field curve shows the usual maximum in the thermal conductivity at about $9 \mathrm{~K}$ with a magnitude of about $1 \mathrm{~W} / \mathrm{cm} \mathrm{K}$. The distinct kink in the zero-field data at about $3 \mathrm{~K}$ is also observed in $\mathrm{CoCl}_{2} \cdot 6 \mathrm{H}_{2} \mathrm{O}$ (Refs. 7 and 8) and must be attributed to magnon-phonon scattering, as will become apparent later on. Owing to this kink the limiting low-temperature behavior is not yet displayed at $1.5 \mathrm{~K}$. From the fact that $\lambda(90 \mathrm{kOe})>\lambda(0)$ we can state immediately that the magnons have a negative influence on the thermal transport, as was already suggested by the kink mentioned above.

It should be noted here that the absolute magnitude of the thermal conductivity depends very much on the quality of the crystals and its history. In general, we observed that a thermal cycle $(4.2$ $\mathrm{K} \rightarrow$ room temperature $\rightarrow 4.2 \mathrm{~K}$ ) as well as a magnetic cycle ( $0 \mathrm{kOe} \rightarrow 90 \mathrm{kOe} \rightarrow 0 \mathrm{kOe}$ ) more or less deteriorates the crystal and causes a reduction of $\lambda$.

The magnetic field dependence of $\lambda$ at a constant temperature was examined in more detail, with the external field along the three principal magnetic axes. The results are shown in Figs. 6, 7, and 8 for the easy, intermediate, and hard direction of spin alignment, respectively. The phase transitions indicated in Fig. 3 are clearly visible, especially when the field is applied parallel to the easy axis. There is a drastic decrease of $\lambda$ at the spinflop critical field $(\sim 8 \mathrm{kOe})$, and at the moment that the paramagnetic phase is reached, $\lambda$ starts to increase monotonically. We observed an increase of $\lambda$ by a factor of 6 in the paramagnetic state (see Fig. 6), but this value is very sensitive to the crystal quality, as can be seen by comparison with Fig. 7. Another interesting feature is the maximum of $\lambda$ in the spin-flop phase. This phenomenon is also observed with $\overrightarrow{\mathbf{H}}$ parallel to the intermediate axis. The relatively smaller field dependence with $\overrightarrow{\mathbf{H}}$ parallel to the hard axis can partly be explained by the smaller $g$ value in that direction, and partly by the number of thermal and 


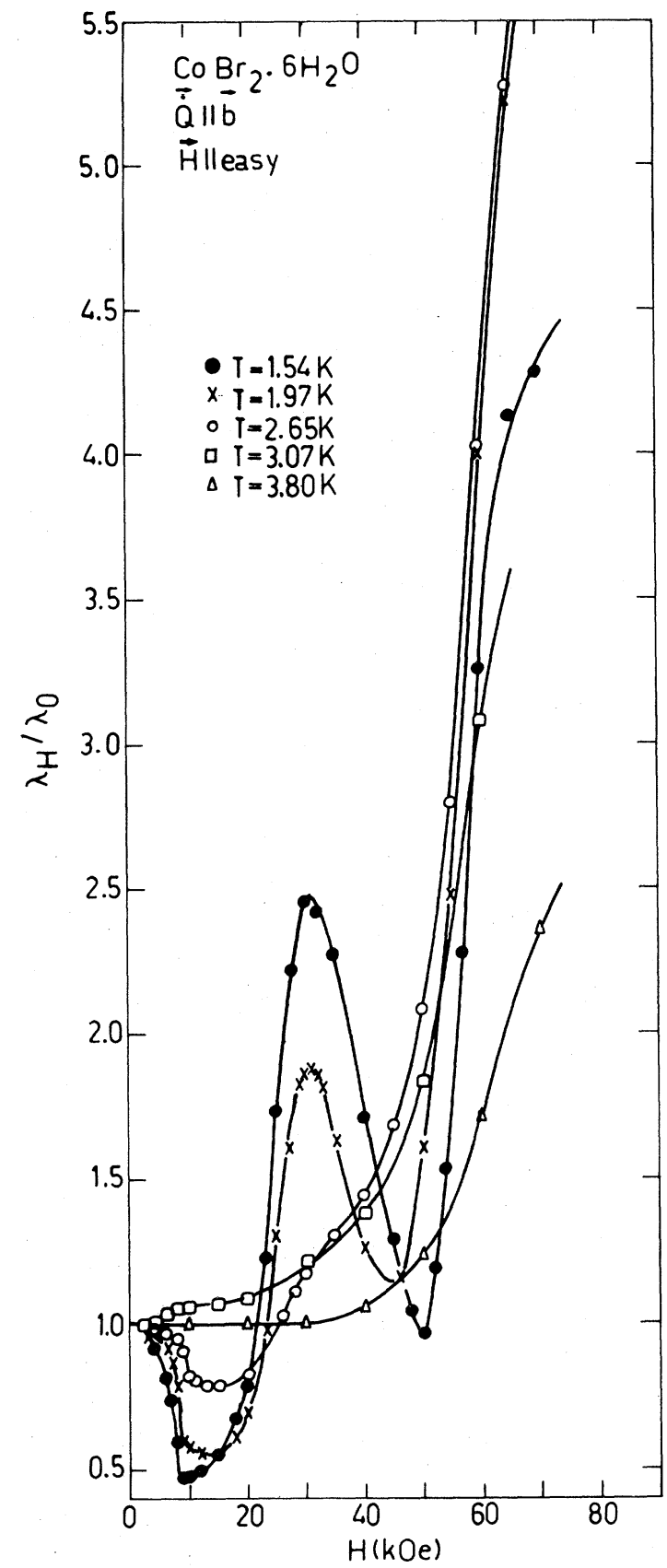

FIG. 6. Field dependence of $\lambda$ in $\mathrm{CoBr}_{2} \cdot 6 \mathrm{H}_{2} \mathrm{O}$ with the field applied in the easy direction, at various temperatures. The results of $\lambda_{H}$ are scaled with respect to the zero-field value $\lambda_{0}$.

magnetic cycles to which the crystal has been subjected.

\section{INTERPRETATION}

We assume that only two kinds of excitations in $\mathrm{CoBr}_{2} \cdot 6 \mathrm{H}_{2} \mathrm{O}$ play a role in the heat-transport pro-

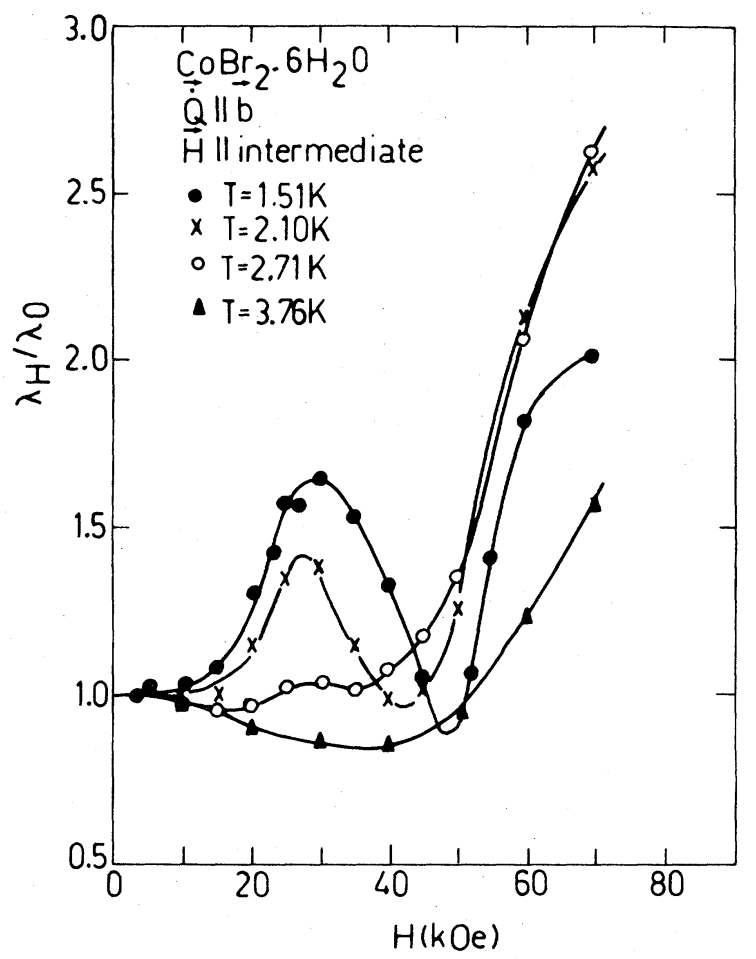

FIG. 7. Field dependence of $\lambda$ in $\mathrm{CoBr}_{2} \cdot 6 \mathrm{H}_{2} \mathrm{O}$ with the external field applied in the intermediate direction.

cess. In the lattice system these excitations are described as phonons; in the magnetic system we can define magnons. This magnon concept is only valid at low temperature $\left(T \lesssim T_{N}\right)$. At higher temperatures the magnetic excitations are more complex and the magnon concept gradually becomes

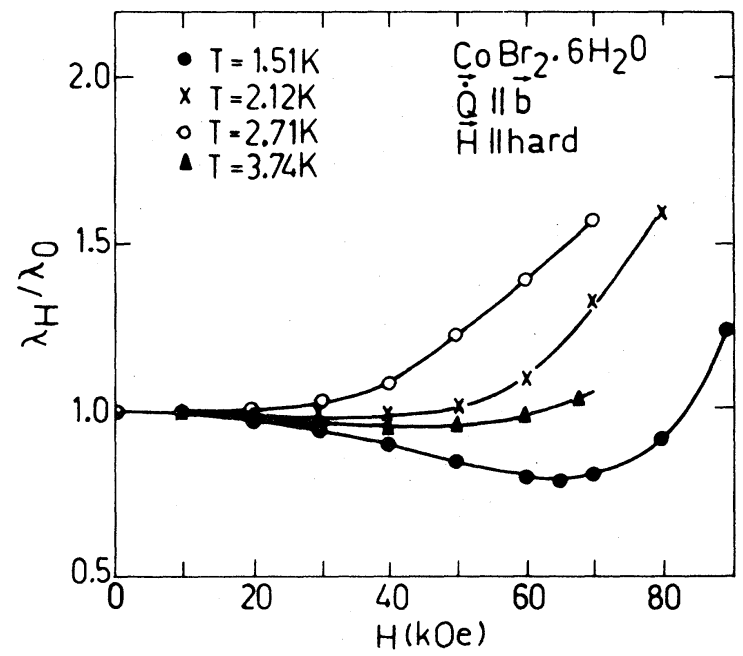

FIG. 8. Field dependence of $\lambda$ in $\mathrm{CoBr}_{2} \cdot 6 \mathrm{H}_{2} \mathrm{O}$ with the external field applied in the hard direction. 
inapt. The phonon system is field independent, so we have to consider the magnon system in order to interpret the field dependence of our results. We follow the linear spin-wave approach as applied by Iwashita et al. ${ }^{9}$ to get expressions for the fielddependent magnon dispersion relations. We shall start with the simplified Hamiltonian:

$$
\begin{aligned}
\mathscr{H}^{\alpha}= & -2 \sum_{\langle i j\rangle}\left(J_{1}^{x} S_{i}^{x} S_{j}^{x}+J_{1}^{y} S_{i}^{y} S_{j}^{y}+J_{1}^{z} S_{i}^{z} S_{j}^{z}\right) \\
& -g^{\alpha} \mu_{B} H^{\alpha}\left(\sum_{i} S_{i}^{\alpha}+\sum_{j} S_{j}^{\alpha}\right) .
\end{aligned}
$$

In this expression $\alpha(=x, y, x)$ denotes the direction of the external field and $i, j$ refers to the sublattices. We have taken a two-dimensional model with only nearest-neighbor interactions. The parameters $J_{1}^{\alpha}$ describe the diagonal exchange interactions in the $a b$ plane (see Fig. 1). For the numerical values of $J_{1}^{\alpha}$ and $g^{\alpha}$ we insert

$$
J_{1}^{x}=-2.28 \mathrm{~K}, g^{x}=4.8,
$$

$$
\begin{aligned}
& J_{1}^{y}=-2.19 \mathrm{~K}, g^{y}=4.8, \\
& J_{1}^{z}=-0.66 \mathrm{~K}, g^{z}=2.2,
\end{aligned}
$$

These values have been somewhat adapted in comparison with Ref. 1 in order to get a fair agreement with the reported critical fields and the observed zero-field antiferromagnetic resonance. ${ }^{4}$

Expressions for the field-dependent dispersion relations with the external field parallel to the easy axis (in our case $x \equiv \gamma$ ) are given in Ref. 9 for the three successive phases. If the field is parallel to the intermediate axis appropriate expressions can be obtained by interchanging $J_{1}^{x}$ and $J_{1}^{y}$ in the expressions for the spin-flop phase. Finally, with the field parallel to the hard axis, the parameters should be changed cyclically $\left(J_{1}^{x} \rightarrow J_{1}^{y}, J_{1}^{y} \rightarrow J_{1}^{z}\right.$, $J_{1}^{z} \rightarrow J_{1}^{x}$ ). In this latter case the value $g=2.2$ is substituted.

The results of our calculations for the field in the easy direction are shown in Fig. 9. There is a
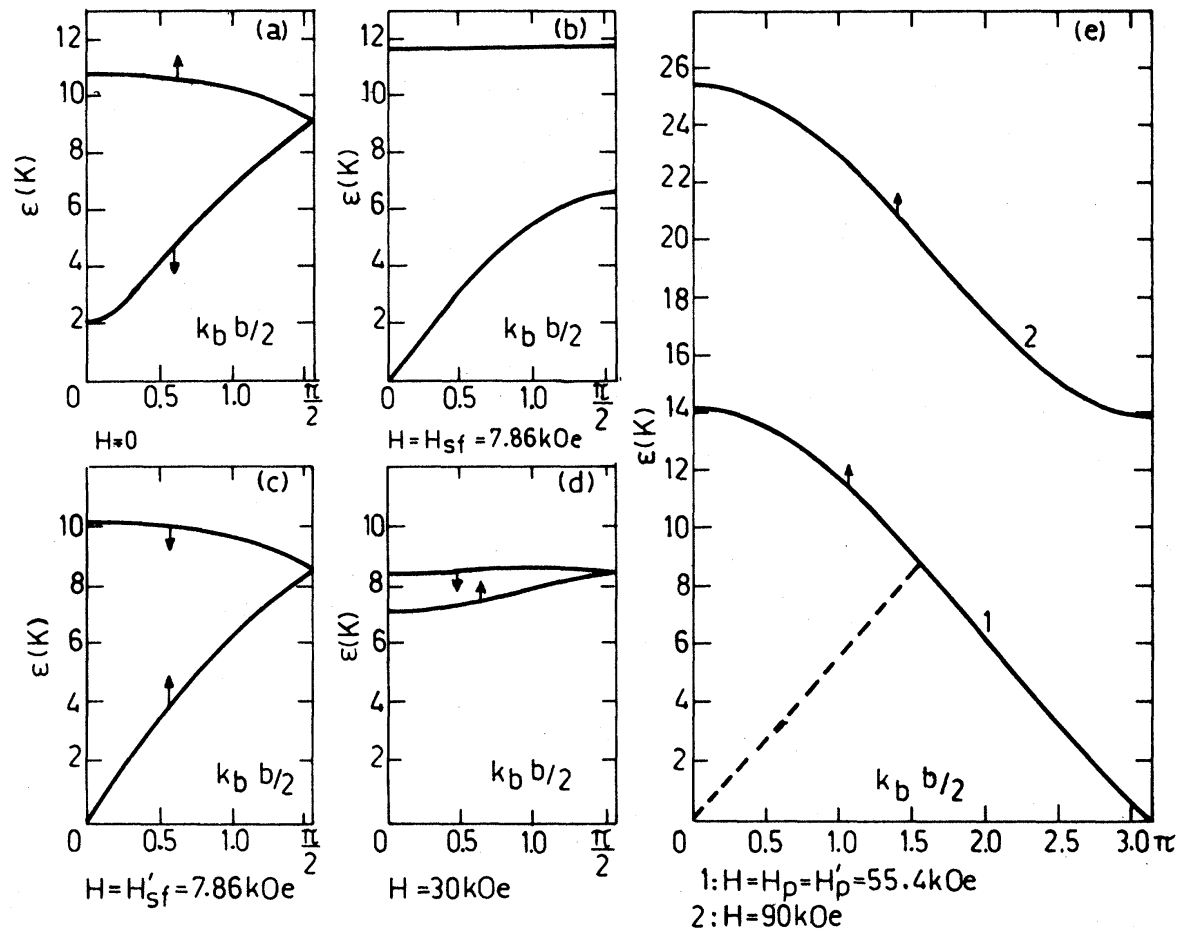

FIG. 9. Spin-wave dispersion of $\mathrm{CoBr}_{2} \cdot 6 \mathrm{H}_{2} \mathrm{O}$ in the $b$ direction, calculated as a function of the external field along the preferred direction. The arrows indicate the shift of the branches when the field is raised. In the antiferromagnetic state the branches shift from the situation of (a) $(0 \mathrm{kOe})$ to $(\mathrm{b})\left(H_{\mathrm{SF}}\right)$. After a discontinuous jump we reach the spinflop phase [(c), $H_{\mathrm{SF}}^{\prime}$ ]. Going from $H_{\mathrm{SF}}^{\prime}$ to $H_{P}$ (the paramagnetic transition field) the two branches gradually change position (d). In the paramagnetic phase the dispersion can be described by only one branch. Raising the field from $H_{P}$ results in a uniform increase of the energies (e). 
discontinuity of the magnon dispersion relation at the spin-flop critical field [Figs. 9(b) and 9(c)]. Furthermore, we note the crossing of the two branches just above $30 \mathrm{kOe}$ and the continuous increase of all mode energies in the paramagnetic state. Since the field dependence of the two branches is rather well reflected by the behavior of the two $k=0$ modes, we show the complete field dependences of these energy gaps in Fig. 10. Inspection of this figure shows that with an external field of $90 \mathrm{kOe}$ applied along the easy direction the lowest-lying magnon mode has an energy of about 14. K. This implies that there will be hardly any magnons at temperatures $T<<14 \mathrm{~K}$ and the thermal transport will be governed by phonons. From Fig. 5 we estimate that, crudely speaking, below $2.5 \mathrm{~K}$ a field of $90 \mathrm{kOe}$ can remove the effect of magnons on the thermal conductivity. As already mentioned above, this effect is negative; the magnons act effectively as a scattering mechanism for the phonons. This fact has been observed before in low-dimensional antiferromagnetic Heisenberg systems $s^{10,11}$ and may possibly be regarded as a general tendency in such compounds, although the experimental evidence is rather restricted.

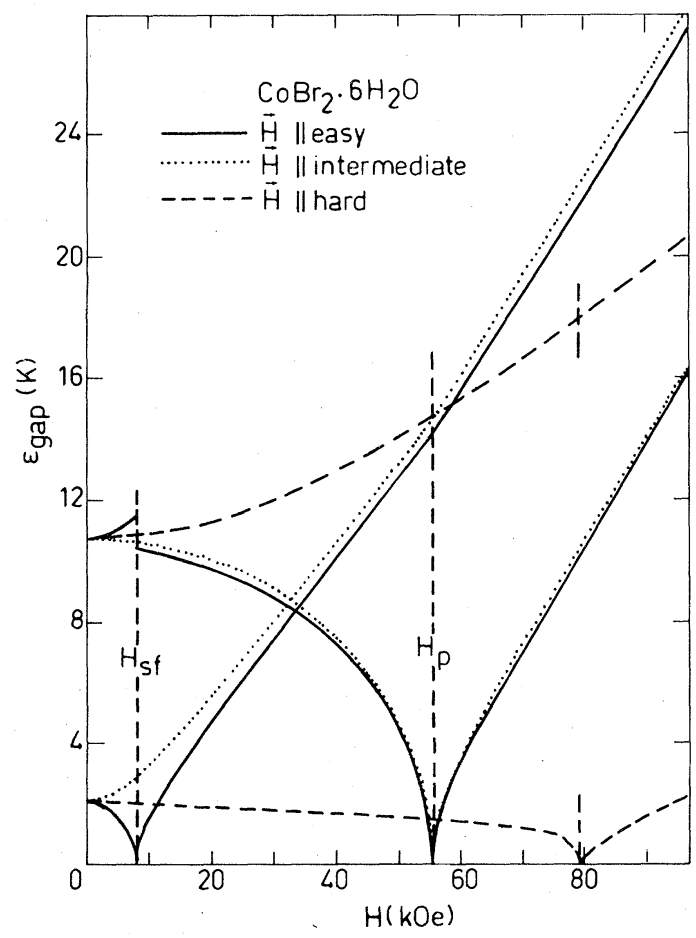

FIG. 10. Field dependence of the two $k=0$ excitations in $\mathrm{CoBr}_{2} \cdot 6 \mathrm{H}_{2} \mathrm{O}$ (the "energy gaps").
We shall now give a qualitative explanation of the observed field dependence of $\lambda$. This explanation is not based on any specific scattering process but was developed after confronting the experimental data with the field dependence of the energy gaps. In the discussion we shall return to this point. The basic assumption of the qualitative interpretation is a direct relation between the density of magnons and the scattering caused by them. In that case a low-lying branch produces more scattering than a higher one and a rising branch results in an increase of $\lambda$, while a lowering branch does the opposite. With the aid of these rules of thumb and Fig. 10 the experimental results of Figs. $6-8$ can be qualitatively understood. The behavior observed at the spin-flop transition and in the paramagnetic state is reproduced very well. The maximum of $\lambda$ in the spin-flop phase (which is also observed with the field applied in the intermediate direction) coincides with the crossing of the two branches at $\sim 33 \mathrm{kOe}$ (see Fig. 10).

In the derivation of the spin-wave spectra no renormalization of the magnon energies has been applied and therefore our interpretation is strictly only valid at $T=0$. The magnon concept itself remains useful throughout the ordered phase, although the critical fields change according to the phase diagram (Fig. 3), which is clearly observed in our measurements. Above the ordering temperature the magnon character of the excitations disappears due to the decreasing correlation lengths. From the experiments we see that this results in a smoothening of the $\lambda-\mathrm{vs}-\overrightarrow{\mathrm{H}}$ curves. The increase of $\lambda$ at high fields is still present.

The interpretation of the experimental data given above does not rely on any detailed knowledge of the actual scattering mechanism involved. Despite this simplification the qualitative interpretation is very satisfactory, especially at the lowest temperatures.

\section{DISCUSSION}

In this section we shall discuss possible processes of magnon-phonon scattering. In the reported temperature range the phonon system can be regarded as fully harmonic, so the most likely processes are those involving only one phonon. The most important processes of this kind are the onephonon-one-magnon resonant interaction and the one-phonon - two-magnon scattering.

The resonant interaction has the largest effect in 
the region where the phonon and magnon dispersion relations intersect. Because of the much larger phonon velocities this intersection occurs at very small wave numbers. The result is a repulsion of the dispersion branches and the generation of magneto-elastic modes. If we restrict ourselves to the phonons, this repulsion in fact creates gaps in the dispersion branches around the energies of the two $k=0$ magnon modes. We performed some numerical calculations on the effect of the resonant interaction on the thermal conductivity, using the same method as Laurence et al. ${ }^{12}$ and assuming that heat is only transported by phonons. Typical results are plotted in Fig. 11. Variation of the interaction parameter, the phonon velocity, or the temperature has a minor effect on the characteristics displayed in this figure. The increase of $\lambda$ in the paramagnetic phase and the maximum in the spin-flop (SF) phase are reproduced correctly. At the critical fields, however, the resonant interaction yields a behavior which essentially differs from the experiments. In contrast to the results plotted in Figs. $6-8$ the calculations show distinct peaks in $\lambda_{H} / \lambda_{0}$ at both $H_{\mathrm{SF}}$ and $H_{p}$. This fact leads us to the conclusion that resonant magnon-phonon interaction cannot be the only process involved. Most likely the two-magnon-one-phonon scattering plays an important role. The effect of this

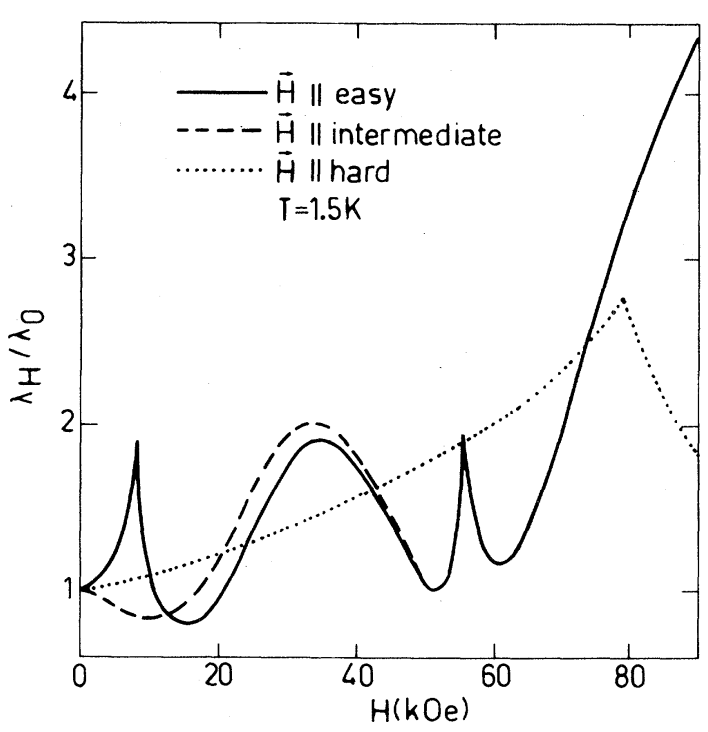

FIG. 11. Calculated field dependence of $\lambda$ in $\mathrm{CoBr}_{2} \cdot 6 \mathrm{H}_{2} \mathrm{O}$ in the case of resonant magnon-phonon interaction. The interaction energy (Ref. 12) amounts to $D_{k}=D \sqrt{\left(k_{b} b / 2\right)}$ with $D=2 \mathrm{~K}$. process on $\lambda$ depends very much on the details of the spin-wave spectrum. Field-dependent thermal conductivities, governed by this three-boson process, have been calculated by $\mathrm{Ono}^{13}$ and Dixon ${ }^{14}$ for the compounds $\mathrm{GdVO}_{4}$ and $\mathrm{MnCl}_{2} \cdot 4 \mathrm{H}_{2} \mathrm{O}$, respectively. Although the spin-wave spectra of these rather isotropic three-dimensional antiferromagnetic Heisenberg systems are quite different from our system, some results seem to be rather general. Especially, the decrease of $\lambda$ at the spinflop critical field and the increase in the paramagnetic phase are reproduced. Whether the twomagnon-one-phonon process also reveals the other observed features of $\lambda$ vs $\vec{H}$ can only be confirmed by a detailed numerical computation, which has not yet been performed. Additional indications about the possible importance of the twomagnon - one-phonon process can be gathered from the temperature dependence of the zero-field data. Dixon and co-workers ${ }^{14(b)}$ observed a slight kink in the $\lambda$-vs- $T$ curve at about $0.8 \mathrm{~K}$, which they attributed to this process. In our case, where we observed some additional scattering around 2.5 $\mathrm{K}$, an analogous plausible explanation can be given. The small $k$ magnons of the lower branch [Fig. 9(a)], which are very numerous, can be scattered by a phonon into a magnon of the upper branch. The phonon involved has an energy of about $9 \mathrm{~K}$. If we assume that the largest contribution to $\lambda$ results from phonons with an energy of $3.8 \mathrm{kT}$ (the maximum of the three-dimensional Debije weight function), it is obvious that at $2.5 \mathrm{~K}$ the heat transport by phonons will be very much suppressed.

As stated above, all the observed characteristics of $\lambda(\overrightarrow{\mathrm{H}})$ and $\lambda(T)$ can be understood in terms of the two-magnon-one-phonon scattering process. This, however, does not exclude other possible scattering processes. Actually, Rives and Bhatia, ${ }^{8}$ who observed a quite similar temperature dependence of the zero-field conductivity in $\mathrm{CoCl}_{2} \cdot 6 \mathrm{H}_{2} \mathrm{O}$, attributed this behavior to a magneto-elastic mode contribution at temperatures below $1 \mathrm{~K}$ in addition to critical phonon scattering above $T_{N}$. One should note, however, that resonant interaction alone cannot account for the observed field dependence. Moreover, critical fluctuations are expected to diverge at $T_{N}$. The smooth behavior of $\lambda_{0}(T)$ around $T_{N}$ therefore seems to suggest other dominating effects. On the other hand, it has been shown that magnons can exist to some extent above $T_{N}$ in low-dimensional compounds. That is why the two-magnon-one- 
phonon scattering possibly can be used to explain the enhanced scattering observed below as well as above $T_{N}$. These arguments are also applicable to the results of $\mathrm{MnCl}_{2} \cdot 4 \mathrm{H}_{2} \mathrm{O} .{ }^{14(\mathrm{~b})}$ The fact that in this case no magnetic effect has been observed in $\lambda(T)$ above $T_{N}$ may reflect the true threedimensional character of this compound.

The scattering activity of the magnons in $\mathrm{MnCl}_{2} \cdot 4 \mathrm{H}_{2} \mathrm{O}$ appears to be much smaller than in $\mathrm{CoBr}_{2} \cdot 6 \mathrm{H}_{2} \mathrm{O}$, as can be deduced from the respective high-field data. This may be caused by the relatively large magnon-phonon coupling in the title compound, which was already anticipated in the Introduction. Crystal-field effects in the $\mathrm{Co}^{2+}$ compound, which are not present in $\mathrm{Mn}^{2+}$ systems, probably are connected with this phenomenon.

In conclusion, we can say that the two- magnon - one-phonon scattering process very likely may explain most of the observed characteristics. Detailed calculations according to Dixon ${ }^{14(a)}$ may confirm this conjecture. Although the qualitative explanation given in the preceding section does not reveal the nature of the scattering process, it appears to be very useful to obtain some insight in the field-dependent behavior of the thermal conductivity of spin-flop systems, even with low dimensionality.

\section{ACKNOWLEDGMENTS}

We thank M. H. M. Dumont for his help in performing the numerical computations and Dr. K. Kopinga for critical reading of the manuscript and some interesting discussions.
1J. W. Metselaar, L .J. de Jongh, and D. de Klerk, Physica 79B, 53 (1975).

${ }^{2}$ K. Kopinga, P. W. M. Borm, and W. J. M. de Jonge, Phys. Rev. B 10, 4690 (1974).

${ }^{3}$ J. N. McElearny, H. Forstat, and P. T. Baily, Phys. Rev. 181, 887 (1969).

${ }^{4}$ T. E. Murray and G. K. Wessel, J. Phys. Soc. Jpn. 24, 738 (1968).

${ }^{5}$ J. A. J. Basten, W. J. M. de Jonge, and E. Frikkee, Phys. Rev. B 21, 4090 (1980).

${ }^{6}$ A. D. Bruce and A. Aharony, Phys. Rev. B 11,478 (1975); E. Callen and H. B. Callen, Phys. Rev. $\underline{129}$, 578 (1963); 139, A455 (1965).

${ }^{7}$ R. H. Donaldson and D. T. Edmonds, Phys. Lett. $\underline{2}$, 130 (1962).

${ }^{8}$ J. E. Rives and S. N. Bhatia, in Magnetism and Magnetic Materials - 1974 (San Francisco), Proceedings of the 20th Annual Conference on Magnetism and Mag- netic Materials, edited by C. D. Graham, G. H. Lander, and J. J. Rhyne (AIP, New York, 1974), p. 174.

${ }^{9}$ T. Iwashita and N. Uryû, J. Phys. Soc. Jpn. $\underline{39}, 36$ (1975). Note: These authors use a $J_{1}$ value that is about half the value we use. They obtain acceptable values for the critical fields by assuming a large $J_{2}$ interaction. This value of $J_{2}$, however, cannot physically be accounted for.

10J. E. Rives, Phys. Lett. A $\underline{36}, 327$ (1971).

${ }^{11}$ J. A. H. M. Buys, J. P. M. Smeets, and W. J. M. de Jonge, J. Magn. Magn. Mater. 15-18, 923 (1980).

${ }^{12} \mathrm{G}$. Laurence and D. Petitgrand, Phys. Rev. B $\underline{8}, 2130$ (1973).

${ }^{13}$ Y. Ono, J. Phys. Soc. Jpn. $\underline{38}, 645$ (1975).

${ }^{14}$ (a) G. S. Dixon, Phys. Rev. B 21, 2851 (1980); (b) G. S. Dixon, V. Benedict, and J. E. Rives, ibid. 21, 2865 (1980). 